\title{
fhSPECT-US Guided Needle Biopsy of Sentinel Lymph Nodes in the Axilla: Is it Feasible?
}

\author{
Aslı Okur ${ }^{1,2,3}$, Christoph Hennersperger ${ }^{1}$, Brent Runyan ${ }^{1}$, José Gardiazabal ${ }^{1,2}$, \\ Matthias Keicher ${ }^{4}$, Stefan Paepke ${ }^{3}$, Thomas Wendler ${ }^{4}$, and Nassir Navab ${ }^{1,5}$ \\ 1 Computer Aided Medical Procedures, Technische Universität München, Germany \\ 2 Department of Nuclear Medicine, Technische Universität München, Germany \\ 3 Department of Gynecology, Technische Universität München, Germany \\ 4 SurgicEye GmbH, Munich, Germany \\ ${ }^{5}$ Computer Aided Medical Procedures, Johns Hopkins University, USA
}

\begin{abstract}
Until now, core needle biopsy of the axillary sentinel lymph nodes in early stage breast cancer patients is not possible, due to the lack of a proper combination of functional and anatomical information. In this work we present the first fully 3D freehand SPECT - ultrasound fusion, combining the advantages of both modalities. By using spatial positioning either with optical or with electromagnetic tracking for the ultrasound probe, and a mini gamma camera as radiation detector for freehand SPECT reconstructions, we investigate the capability of the introduced multi-model imaging system, where we compare both 3D freehand SPECT and $3 \mathrm{D}$ ultrasound to ground truth for a realistic breast mimicking phantom and further analyze the effect of tissue deformation by ultrasound. Finally, we also show its application in a real clinical setting.
\end{abstract}

\section{Introduction}

Sentinel Lymph Node Biopsy (SLNB) is a standard surgical procedure in early breast cancer for determination of the cancer stage 11. By injecting dedicated radiotracers (commonly a Tc-99m-colloid) close to the tumor, and using appropriate imaging techniques with dedicated gamma radiation detectors, such as scintigraphy or SPECT, it is possible to visualize the sentinel lymph node(s) $(\mathrm{SLN}(\mathrm{s}))$, which is/are the first node on the lymphatic drainage way and is/are most likely to have (micro-)metastasis in case the tumor has progressed.

During surgery, the breast surgeon can differentiate between the SLNs and non-radioactive lymph nodes (LNs) with small hand-held radiation detectors, called gamma probes. However, this is not an easy task, especially in patients where the SLN is too close to the actual injection site due to the collimation and sensitivity of the gamma probe as well as the shine-through and shadowing effect of the injection. It has been shown that freehand SPECT (fhSPECT) 2], an intra-operative 3D nuclear imaging technique based on tracked gamma probes, can improve the discrimination for breast cancer patients 3 . Furthermore, mini gamma cameras, providing realtime $2 \mathrm{D}$ images of the axilla, recently also have found their way into the operating room 4 .

P. Golland et al. (Eds.): MICCAI 2014, Part I, LNCS 8673, pp. 577-584, 2014.

(C) Springer International Publishing Switzerland 2014 
Until now, these solutions made the localization of the SLNs much more convenient for the surgeon, however, the excision of the SLN still takes place inside the operating room. The reason for this fact is that such nuclear images alone do only provide functional, but no anatomical information.

In contrast to this, US imaging is providing anatomical information and is also common practice for staging of lymph nodes. However, US can only detect metastatic LNs since these LNs are comparably bigger and appear conspicious in US images. In early stage breast cancer where the SLNB is indicated, metastasic and healthy LNs in axilla cannot be differentiated in US.

In order to be able to perform US-guided core needle biopsies of the radioactively labeled SLNs, one needs to combine the advantages of both modalities. The fusion of fhSPECT with US could thus help the physician to identify the SLNs on US images and eventually to perform core needle biopsies during US examination by using only local anesthesia.

In general, combining US with nuclear information has been proposed by [5], where optically tracked gamma probes were used, but did not include 3D SPECT-like reconstructions. Nowadays, there are solutions available, which allow quasi real-time fusion of 3D nuclear functional images with the US. Freesmeyer et al. [6] recently published their first clinical experience and results with such a system on thyroid patients. However, this work lacks on quantitave evaluation of the quality of the $3 \mathrm{D}$ reconstructions and uses a non-diagnostic version of fhSPECT (lower image resolution and sensitivity).

In this work, we investigate for the first time the fusion of the fhSPECT and US data for the application in breast cancer. To do so, we propose a system using optically tracked 3D fhSPECT based on gamma cameras instead of probes to increase image quality, and 3D freehand US data either with optical (OP) or electromagnetic (EM) tracking. We compare both to ground truth SPECT/CT obtained for a realistic breast mimicking phantom and analyze the effect of tissue deformation by US. Finally we also show results of such a system on real patients.

\section{Materials and Methods}

Hardware Setup. For our study we used a Sonix RP US system (Ultrasonix, MA, USA) with an linear US probe (L14-5 GPS) (Fig. 1(a)), which incorporates a sensor for the EM tracking system (3D Guidance driveBAY 2, Ascension Technology Corporation, VT, USA). To compare the accuracy of the EM to the optical tracking for US, we mount a reference target on the probe (Fig. 1(b)).

The fhSPECT images are generated using a declipseSPECT Imaging Probe system (SurgicEye GmbH, Munich, Germany), which uses a 2D mini gamma camera (Crystal Photonics, Berlin, Germany) for radiation detection (Fig. 1(c)) and an infrared optical tracking system (Polaris Vicra, Northern Digital Inc., Ontario, Canada) for spatial positioning. The 3D fhSPECT images are reconstructed using the declipseSPECT software. The US images and the meta data with the US settings are saved synchronized with the tracking information from both of the localization systems. 

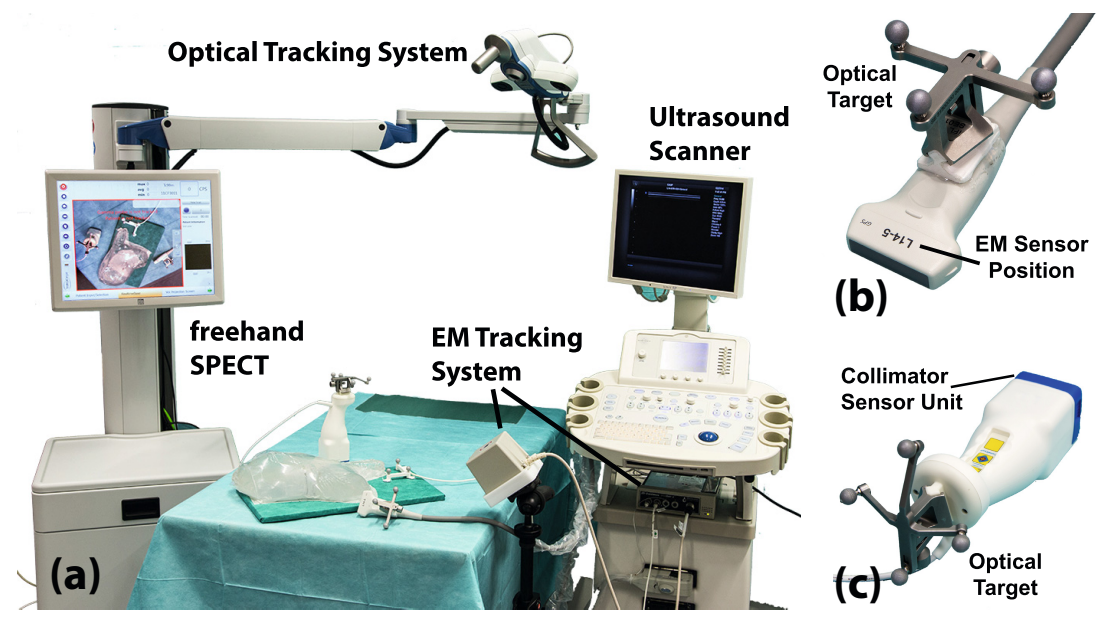

Fig. 1. (a) Hardware setup. (b) Ultrasound probe. (c) Mini gamma camera.

fhSPECT Reconstruction. The fhSPECT system uses position information from the optical tracking system in addition to nuclear information from the attached radiation detector, in our case the mini gamma camera. This information is then combined with calibration data specific for the detector to produce an input data set. The input data set is then processed using a standard Maximum Likelihood Expectation Maximization (MLEM) solver. Post processing options can include Gaussian filtering, if desired.

US Calibration. To calibrate the US probe for both tracking systems, a calibration phantom similar to the one in [7], is constructed. It consists of a pyramidal aluminum frame, where its three faces are covered with a thin nylon layer. In contrast to [7], the three faces all join at the same vertex with face angles of $90^{\circ}$, resulting in a trirectangular tetrahedron, whose three edges form a local coordinate system. For calibration, an US sequence of the pyramid is acquired such that the intersection of the US image plane and the pyramid forms a triangle. Based on the pyramid coordinate system, direct point correspondences from 2D image points in the US plane to the corresponding positions in $3 \mathrm{D}$ space can be established, and used to obtain the transformation from the US plane to the coordinates of the tracking system, cf. [8].

US Compounding. As vizualization is of crucial importance for the fusion of fhSPECT to 3D ultrasound data, we employ an volume compounding step, i.e. an interpolation of the acquired, irregularly sampled US data on a rectangular grid, yielding a 3D volume. Following [9], we apply compounding as a backwardwarping normalized convolution directly on the ultrasound scanlines. As this system provides fast compounding and resulting volumes of high quality, it is suitable for direct fusion with fhSPECT images. 
fhSPECT-US Fusion. The fusion of the final images for both fhSPECT and US data is assured with the aid of a reference target, which is tracked both optically and electomagnetically, cf. Fig. 2(a). The final DICOM files generated by the fhSPECT system are saved in the coordinate system of the optical reference target on the patient. Therefore, we use this coordinate system as the base for our calculations. For a point in US plane, in the case of dual tracking, we apply the following transformation chain to get its positions in the reference frame of the optical reference target

${ }^{r e f O P} \mathbf{T}_{U S}={ }^{\text {refOP }} \mathbf{T}_{\text {refEM }} \cdot{ }^{\text {refEM }} \mathbf{T}_{\text {worldEM }} \cdot{ }^{\text {worldEM }} \mathbf{T}_{\text {probe } M M} \cdot{ }^{\text {probeEM}} \mathbf{T}_{U S}$

In the case of optical tracking only, the sequence shortens to

$$
{ }^{\text {refOP }}{ }^{\mathbf{T}_{U S}}={ }^{\text {refOP }} \mathbf{T}_{\text {worldOP }} \cdot{ }^{\text {world } O P} \mathbf{T}_{\text {probeOP }} \cdot{ }^{\text {probeOP }} \mathbf{T}_{U S},
$$

where the transformations from the US plane to the optical target ${ }^{\text {probeOP }} \mathbf{T}_{U S}$, as well as to the EM sensor on the US probe ${ }^{p r o b e E M} \mathbf{T}_{U S}$ are calculated as mentioned before. Furthermore, the transformation between the optical tracking target, serving as a reference for the patient, and the electromagnetic sensor rigidly attached to it, is known from precision manufactured target construction files. Alternatively, a calibration of the reference target w.r.t. EM and optical system can be achieved by hand-eye calibration [8].

\section{Experiments and Results}

Phantom Design. In order to demonstrate the technical feasibility of the system, possible phantom designs were discussed with experienced breast surgeons and nuclear medicine physicians to model the clinical scenario in the best possible way. With the goal of a realistic biopsy phantom for the axilla region, we cut from a plastic female torso mannequin the region of one breast and the axilla and use this as a mold for a phantom. As tissue mimicking material, Ceraflex N530 gel (C Tromm GmbH, Germany) is used, as it is well suited for US, SPECT and CT imaging, and additionally deforms in a realistic way when pressure is applied to the surface (Fig. 2(a)). Thus the phantom does not only allow for an evaluation of the US-SPECT fusion by itself, but also for an analysis of the changes in accuracies due to tissue deformation.

To evaluate the fusion scenario with the goal of finding SLNs to biopsy, 4 spheres with similar sizes as the SLNs (240-300 ml) are placed in the phantom. One of spheres is filled with $1.1 \mathrm{MBq}$ of Tc-99m before insertion into the phantom to represent a SLN, while the others are filled with water representing nonradioactive LNs in the same region.

Data Acquisition. We scanned our phantom with both the fhSPECT and the 3D US system in 5 different configurations with varying positions and orientations of one or both tracking systems w.r.t the breast phantom (cf. Fig. 1). In each configuration, we scanned the phantom at least once with fhSPECT for 

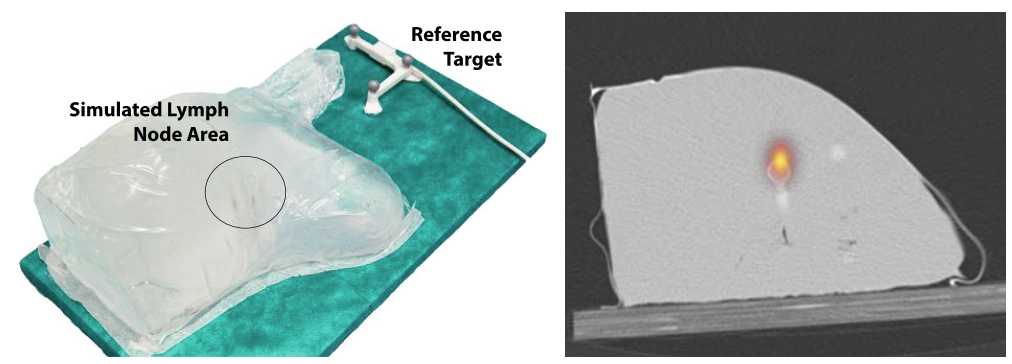

Fig. 2. (a) Tissue mimicking breast phantom, where the spheres represent LNs. (b) $\mathrm{SPECT} / \mathrm{CT}$ fusion of the radioactive sphere in the phantom.

about 2 minutes. Furthermore, at least 2 sweeps were acquired with the 3D US system for each configuration, trying to cover all spheres. To evaluate the quality of the 3D US system on its own, both EM and OP tracking systems were used simultaneously for the acquistions, enabling a direct comparison of the resulting accuracies for both systems. Furthermore, the impact of deformation changes was evaluated separately using 4 US acquisitions with varying pressure applied to the breast phantom surface.

All phantom acquisitions for both fhSPECT and US were performed by the same person to avoid user-dependent variation. Finally, a SPECT/CT (Siemens Symbia T6) of the phantom was acquired to enable direct comparison of the acquired data ground truth image data (Fig. 2 (b)).

Offline Evaluation. We evaluated the fhSPECT and US images separately to the CT, by comparing the centroids of the spheres placed in the phantom w.r.t. the optical reference target. For this, we first calculated the transformation from CT coordinates to the reference target coordinates by the means of point based registration. This is achieved by using the coordinates of the centers of the retroreflective spheres on the reference target in the CT image and their corresponding known coordinates based on its CAD design. The RMS registration error for our transformation was calculated as $0.8 \mathrm{~mm}$.

For each of the fhSPECT reconstructions we then segmented the hotspot with an automatic region growing algorithm using the global maximum as the seed and $50 \%$ as threshold. Later, we computed the centroid of the segmented hotspot and calculated its coordinates w.r.t. the patient reference frame using the meta information of the DICOM tags of the reconstruction.

The online reconstructions with $3 \mathrm{~mm}$ voxel size resulted in $2.17 \mathrm{~mm}$ average localization error (ALE) (stdev: $1.04 \mathrm{~mm}$ ) for fhSPECT reconstructions. We additionally reconstructed the same acquisitions with $1 \mathrm{~mm}$ voxelsize for the same volume. These had an average error of $1.73 \mathrm{~mm}$ (stdev: $0.67 \mathrm{~mm}$ ) instead. As a reference for fhSPECT quality, we calculated the fusion error between SPECT and CT centroids as $3.5 \mathrm{~mm}$, cf. Fig 2(b).

For all US scans, we manually segmented the spheres in the acquired 3D US scans for both optical and EM tracking. Average localization errors of the sphere 


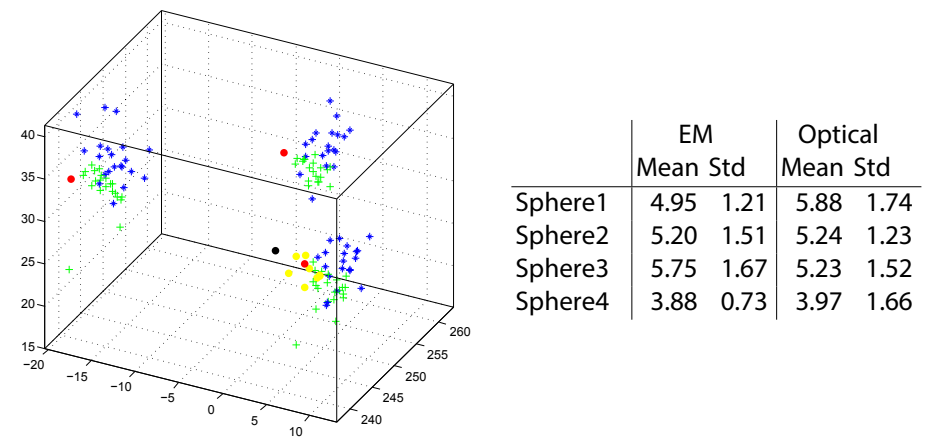

Fig. 3. (a) Segmented sphere centroids compared for ground truth CT (red), fhSPECT (yellow), US EM (blue) and US optical (green). (b) Localization errors in mm calculated for US and ground truth (CT).
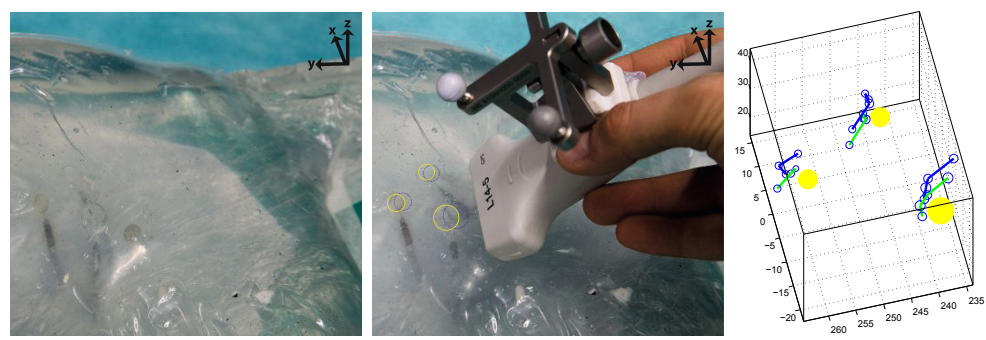

Fig. 4. Effect of deformation on phantom. Phantom position as used for fhSPECT (a) and Ultrasound (b) show noticeable deformation (marked circles). (c) Extracted centroids for trajectories with increasing pressure for US EM (blue) and US OP (green) in relation to the $\mathrm{CT}$ (yellow).

centroids from 3D-US to ground truth yielded $5.36 \mathrm{~mm}$ (stdev: $1.56 \mathrm{~mm}$ ) for optical and $5.22 \mathrm{~mm}$ (stdev: $1.49 \mathrm{~mm}$ ) for EM tracking respecetively. Results for the average errors to the 4 individual spheres are given in Fig. 3 . From the 3D visualization, a systematic error of both tracking systems in direction of the US probe orientation can be observed. We also evaluated the deviations from ground truth regarding varying pressure applied during acquisition. Exactly this pressure was found to be the main cause for localization errors, as with increased pressure, the errors increase similarily for both tracking systems due to higher deformation (mean ALE $5.16 \mathrm{~mm}$ for normal pressure, $10.46 \mathrm{~mm}$ for high pressure), cf. Fig. 4.

Patient Studies. We also scanned 7 patients preoperatively right after radiotracer injection and scintigraphy imaging. Since our system with optical tracking of the US probe is not yet clinically approved, we used a GE Logiq E9 US system (GE Milwaukee, USA) with built-in EM tracking instead, which is the same as our previous setup. This US system can also visualize on-site the imported 3D fhSPECT DICOM images fused on the 2D image plane of the tracked US probe. 

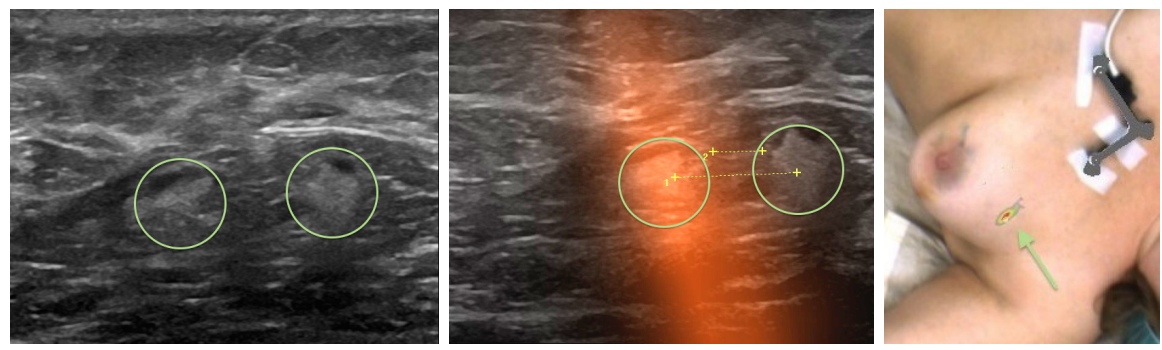

Fig. 5. (a) US image of two LNs in the axilla of the patient. (b) Fused image of fhSPECT and US. Left LN is identified as the sentinel. The distance of the two LNs is from centers $11.7 \mathrm{~mm}$ (1), from borders $4.7 \mathrm{~mm}$ (2). (c) fhSPECT reconstruction overlaid on the patient.

In order to experience the clinical challenges posed by an interventional usage of such a fused fhSPECT-US system, we scanned one patient additionally once more before surgery inside the OR. (Fig. 5). The accuracy was enough for the surgeon to determine the SLN on US images and he was confident that he would be even able to perform the fhSPECT-US-guided core needle biopsy, if approved.

\section{Discussion}

Although fhSPECT has much smaller detector sizes than the conventional SPECT, it achieved better localization scores. We believe that this is due to smaller distances between the actual radiation source and the detector and therefore provides better detection statistics with the mini gamma camera. Another reason could be its smaller voxel size ( $1 \mathrm{~mm}$ for fhSPECT, $4.8 \mathrm{~mm}$ for SPECT).

The localization error for US is considered to be mainly due to the deformation of the phantom during scanning. This is crucial for the fusion of the two images in real scenarios, as small movements of the patient might easily interfere with the reliability of the fusion. In an intervention using local anestesia this can easily happen between two scans and needs to be taken into account. However, new scanning protocols can be suggested for compensation of the deformation effect, such as a preceding scan without any pressure applied as reference, prior to the actual US scan.

For US, both tracking systems achieved comparable scores, where both have their own advantages and disadvantages. EM provides easier handling of the US probe than the optical tracking and therefore, final solutions using EM tracking might ease the acceptance by the end users. However, since the fhSPECT system already uses optical tracking and integrated augmented reality visualization, optical tracking of the US probe might be beneficial in final fhSPECT-US solutions in terms of visualization and integration into one system.

Despite the deformation of soft tissue, results were satisfactory in regard to the clinical application towards fhSPECT-US guided core needle biopsies of SLNs. In our patient studies, we could identify one LN to be the sentinel on the fused 
images. However, to clinically approve this, an interventional study, investigating the patient outcome by comparison of radio-guided surgical SLNBs and fhSPECT-US guided core needle SLNBs is essential.

\section{Conclusion}

In this work we presented the first fully 3D fhSPECT-US fusion using mini gamma cameras and optional EM or optical tracking. Our evaluation showed that both optical and EM tracking systems are suitable for such multi-modal data fusion within clinical settings. This might allow actual needle biopsies of SLNs in the future and reduce the number of comparably invasive SLNB procedures.

Acknowledgements. We would like to thank the Bayerische Forschungsstiftung (Project RoBildOR) and SurgicEye GmbH.

\section{References}

1. Vidal-Sicart, S., Olmos, R.V.: Sentinel node mapping for breast cancer: Current situation. Journal of Oncology 2012, e361341 (2012)

2. Wendler, T., Herrmann, K., Schnelzer, A., Lasser, T., Traub, J., Kutter, O., Ehlerding, A., Scheidhauer, K., Schuster, T., Kiechle, M., Schwaiger, M., Navab, N., Ziegler, S.I., Buck, A.K.: First demonstration of 3-D lymphatic mapping in breast cancer using freehand SPECT. EJNMMI 37(8), 1452-1461 (2010)

3. Bluemel, C., Schnelzer, A., Okur, A., Ehlerding, A., Paepke, S., Scheidhauer, K., Kiechle, M.: Freehand SPECT for image-guided sentinel lymph node biopsy in breast cancer. EJNMMI 40(11), 1656-1661 (2013)

4. Bricou, A., Duval, M.-A., Charon, Y., Barranger, E.: Mobile gamma cameras in breast cancer care - a review. EJSO 39(5), 409-416 (2013)

5. Wendler, T., Feuerstein, M., Traub, J., Lasser, T., Vogel, J., Daghighian, F., Ziegler, S.I., Navab, N.: Real-time fusion of ultrasound and gamma probe for navigated localization of liver metastases. In: Ayache, N., Ourselin, S., Maeder, A. (eds.) MICCAI 2007, Part II. LNCS, vol. 4792, pp. 252-260. Springer, Heidelberg (2007)

6. Freesmeyer, M., Opfermann, T., Winkens, T.: Hybrid integration of real-time US and freehand SPECT: proof of concept in patients with thyroid diseases. Radiology, 132-415 (January 2014)

7. Liu, J., Gao, X., Zhang, Z., Gao, S., Zhou, J.: A new calibration method in 3d ultrasonic imaging system. In: Proc. of the 20th Ann. Int. Conf. of the IEEE Engineering in Medicine and Biology Society, vol. 2, pp. 839-841. IEEE (1998)

8. Tsai, R.Y., Lenz, R.K.: A new technique for fully autonomous and efficient 3D robotics hand/eye calibration. IEEE Transactions on Robotics and Automation 5(3), 345-358 (1989)

9. Hennersperger, C., Karamalis, A., Navab, N.: Vascular 3D+T freehand ultrasound using correlation of doppler and pulse-oximetry data. In: Stoyanov, D., Collins, D.L., Sakuma, I., Abolmaesumi, P., Jannin, P. (eds.) IPCAI 2014. LNCS, vol. 8498, pp. 68-77. Springer, Heidelberg (2014) 